\title{
Phenolic compounds in mono-cultivar extra virgin olive oils from Algeria
}

\author{
By M. Douzane ${ }^{1}$, A. Tamendjari ${ }^{3, *}$, A.K. Abdi', M-S. Daas ${ }^{1}$, F. Mehdid $^{1}$ and M.M. Bellal ${ }^{2}$ \\ ${ }^{1}$ Laboratory of Food Technology, National Institute of the Agronomic research of Algeria. CRP Mehdi \\ Boualem. P O BOXE 37 road of Baraki. Algiers-Algeria \\ ${ }^{2}$ Department of Technology of the Food industry and human Nutrition. Agronomic National Institute \\ of Algiers (INA). El Harrach. Algiers-Algeria \\ ${ }^{3}$ Laboratory of Applied Biochemistry, Faculty of Natural and Life Sciences, University of Bejaia, Algeria \\ * Corresponding author: Abderezakt@yahoo.fr
}

\begin{abstract}
RESUMEN
Componentes fenólicos en aceites de oliva vírgenes monovarietales de Argelia

La alta estabilidad oxidativa de los aceites de oliva vírgenes está relacionada con su alta relación de ácidos grasos monoinsaturados/poliinsaturados y con la presencia de componentes antioxidantes, como tocoferoles y polifenoles. En este trabajo se llevó acabo un aislamiento y cuantificación de compuestos fenólicos de aceites de oliva virgenes de variedades argelinas, con el fin de conocer sus potenciales usos y beneficios. La cuantificación de los componentes fenólicos y o-difenólicos se realizó por método tradicional de Folin-Ciocalteu y mediante la reacción con molibdato de sodio, respectivamente. Los compuestos fenólicos individuales cualitativos y cuantitativos en los extractos y en una mezcla estándar fueron determinados por HPLC.

Los resultados experimentales muestran que el tirosol, hidroxitirosol y el 4-HBA (ácido 4-hidroxibenzoico) son los principales compuestos fenólicos individuales identificados en el conjunto de la población de las variedades estudiadas.

Por otra parte, la población de las variedades con frutos grandes (Grosse du Hamma, Aghenfas, Azeradj, Aguenaou, Aberkane, Bouchouk de Guergour, X-Aghenfas, Rougette de Guelma, Sigoise) se distinguen claramente de las variedades con frutos medios y pequeños. Además, la población de variedades con frutos pequeños (Hamra, Chemlal, Boughenfas, Limli, Aimel y Mekki) presentó el nivel más alto en oleuropeína.
\end{abstract}

PALABRAS CLAVE: Aceite de oliva virgen - Composición química - Compuestos fenólicos - Variedades argelinas.

\section{SUMMARY}

Phenolic compounds in monocultivar extra virgin olive oils from Algeria

The high oxidative stability of virgin olive oil is related to its high monounsaturated/polyunsaturated ratio and to the presence of antioxidant compounds, such as tocopherols and phenols. In this paper, the isolation of phenolic compounds from virgin olive oil from several Algerian varieties was tested and discussed in order to know its potential uses and benefits. Quantification of phenolic and o-diphenolic substances was performed using the traditional Folin-Ciocalteau method and the sodium molybdate reaction, respectively. The qualitative and quantitative characterizations of phenolic compounds were carried out by HPLC.
The experimental results show that tyrosol, hydroxytyrosol and 4-HBA (4-hydroxybenzoic acid) are the main individual phenolic compounds identified in the varieties studied. The varieties with large fruits (Grosse du Hamma, Aghenfas, Azeradj, Aguenaou, Aberkane, Bouchouk de Guergour, $X$-Aghenfas, Rougette de Guelma, Sigoise) are clearly distinguished from the varieties with medium-sized and small fruits by having the highest levels of individual phenolic compounds. Moreover, varieties with small fruits (Hamra, Chemlal, Boughenfas, Limli, Aimel and Mekki) presented the highest levels of oleuropein.

KEY-WORDS: Algerian varieties - Chemical composition - Phenolic compounds - Virgin olive oil.

\section{INTRODUCTION}

Olive oil is obtained from the fruit of olive trees (Olea europaea L.) and is a genuine fruit juice with excellent nutritional, sensorial and functional quality. It represents a typical lipid source of the Mediterranean diet, whose consumption has been associated with a low incidence of cardiovascular diseases, neurological disorders, breast and colon cancers, as well as hipolipidemic and antioxidant properties (Gemino et al., 2002). Some of these effects are associated with extra virgin olive oil's contents of phenolic compounds and tocopherols and high amounts of oleic acid. The amount and composition of phenolic compounds in virgin olive oil depend on several factors such as olive cultivar, degree of maturation and agronomic and technological aspects of production (Forcadell et al., 1987; Saitta et al., 2002; Pinelli et al., 2003 and Van der Sluis 2005). Phenolic compound content is an important parameter for the evaluation of virgin olive oil quality as phenols largely contribute to oil flavor (Gutiérrez-Rosales et al. 2003) as long as it is protected from auto oxidation (Pellegrini et al., 2001; Mateos et al., 2005). Virgin olive oil contains a large number of phenolic compounds including phenyl alcohols, such as tyrosol, hydroxytyrosol, phenolic acids, flavonoids, as well as other more complex secoiridoid derivatives from oleuropein and ligstroside (Montedoro et al., 1992, Tsimidou 
et al., 1992; Angerosa et al., 1995, Cortesi et al., 1995, Pinelli et al., 2003, Artajo et al., 2007).

In this paper, HPLC was used to evaluate the qualitative and quantitative composition of the phenolic compounds in Algerian olive oils with the aim to identify new compounds and to evaluate differences among samples obtained from various olive varieties (twenty one mono-cultivars).

\section{MATERIALS AND METHODS}

\subsection{Plant material}

The present work was carried out on monovarietal virgin olive oils from twenty-one Algerian cultivars: Chemlal (Che), Azeradj (Azj) and Sigoise (Sg) (they are main populations varieties of the national olive-growing orchard), Mekki (Mk), Neb djemel (Nd), Hamra (Ham), Blanquette de Guelma (Blg), Aberkane (Abr), Aimel (Ai), Rougette de la Mitidja (Rm), Aguenaou (Agn), Boughenfas (Bgf), Bouichret (Bcht), Aghenfas (Agf), Bouchouk de Guergour (Bkg), X-Aghenfas (X-Agf), Bounguergueb (Bng), Ronde de Miliana (Rdm), Limli (Lim), Grosse du Hamma (Grh), Rougette de Guelma (Rg) (They are local varieties, the least cultivated).

The olive fruits used were hand-picked from mature trees (five per variety) in two crop years. They were obtained from the experimental farm of the technical institute of fruit-bearing arboriculture and the vine (TIFAV). This farm is located $30 \mathrm{~km}$ from Algiers in the Birtouta district, precisely on the Mitidja plain at the piedmont on the south side of Sahel's hills with a plane relief. This area presents an arboricultural vocation, it is characterized by a Mediterranean climate on sub-wet bioclimatic floor, with mild, rainy winters and hot summers. It is situated $50 \mathrm{~m}$ above sea level at $35^{\circ} .55$ latitude and at $2^{\circ} .55$ longitude, east. The olivegrowing orchards have 34 varieties, of which 9 are of foreign origin. The soil is alluvial (clay, fine and coarse silts) favorable for good water retention.

The samples were collected on the same day at the black-ripe stage. At full maturity, before natural fruit drop, the skin is black and the fruit removal force is low.

Each sample consisted of approximately $6 \mathrm{~kg}$ of olives, which were taken exclusively from the five trees. The olives were picked at shoulder height from all around the canopy and transferred quickly to the laboratory in plastic crates.

\subsection{Oil extraction}

Oil was extracted from the whole olive fruit in an oleodoseur Rappenelli type laboratory mill, equipped with a centrifuge divider (3000 r.p.m.), in the following stages: washing, grinding, kneading and centrifugation. After the grinding of olives, the mixture was treated with water at $28^{\circ} \mathrm{C}(60 \mathrm{~mL}$ for $920 \mathrm{~g}$ of olive paste) and centrifuged, the mixture was then kneaded for 15 min on cold and 15 min on hot. The oil was recovered after decantation in order to eliminate the vegetal water. The extracted oil was kept in a dark brown flask at $4^{\circ} \mathrm{C}$.

\subsection{Analytical indices}

Free acidity, peroxide value and UV spectrophotometric indices (at 232 and $270 \mathrm{~nm}$ ) were determined according to the European Community's official methods (Official Journal of the European Community, 1991).

\subsection{Extraction of the phenolic fraction}

Extraction was carried out according the conditions described by Bendini et al. (2003), an octyl-bonded phase cartridge, $\mathrm{C}_{8}$-Sep-Pak vac $\mathrm{C}_{8}$ 3cc $(500 \mathrm{mg})$, was placed in a vacuum elution apparatus, washed with $6 \mathrm{~mL}$ of $n$-hexane and then conditioned with $6 \mathrm{~mL}$ of acetonitrile. One gram of oil, dissolved in $6 \mathrm{~mL}$ of $n$-hexane was passed through the cartridge in order to remove the non-polar fraction of the oil. The polar fraction was consecutively eluted with $6 \mathrm{~mL}$ of acetonitrile, $6 \mathrm{~mL}$ of methanol and then with $6 \mathrm{~mL}$ of $\mathrm{CH}_{3} \mathrm{OH}$ water $(1: 1, \mathrm{v} / \mathrm{v})$. The fractions were combined and evaporated. After extraction, the residues were dissolved in $1.0 \mathrm{~mL}$ of $\mathrm{CH}_{3} \mathrm{OH}$-water $(1: 1, \mathrm{v} / \mathrm{v})$ and filtered through a $0.45-\mu \mathrm{m}$ nylon filter.

\subsection{Colorimetric determination of total phenols}

The total phenol contents of the extracts was determined by the Folin-Ciocalteau spectrophotometric method at $750 \mathrm{~nm}$, using a gallic acid calibration curve (Singleton and Rossi, 1965).

\subsection{Colorimetric determination of O-diphenols}

$0.5 \mathrm{~mL}$ of phenolic extract obtained from the olive oil using the SPE method was evaporated, the residue was then dissolved in $5 \mathrm{~mL}$ of methanol/ water (1:1), and the solution was filtered. A mixture of $4 \mathrm{~mL}$ of the solution with $1 \mathrm{~mL}$ of a $5 \%$ solution of sodium molybdate dihydrate in ethanol/water (1:1) was shaken vigorously. After $15 \mathrm{~min}$, the absorbance was measured at $370 \mathrm{~nm}$ using a gallic acid calibration curve (Mateos et al., 2001).

\subsection{Preparation of reference compounds (standard phenolic)}

The following commercial products were used: gallic acid (GalA) was from Sigma, protocatechuic acid (PA), 3,4-di hydroxyphénylethanol acid (3,4-DHPEA) or hydroxytyrosol, tyrosol (Tyr), 4-hydroxybenzoïc acid (4-HBA), caffeic acid (CafA), vanillic acid (VA), $p$-coumaric acid ( $p$-CA), ferulic acid (FA) or trans-4-hydroxy-3-methoxycinnamic 
acid, o-coumaric acid (o-CA), 3-methoxybenzoïc acid (3-MBA), and cinnamic acid (CinA) were from Fluka (Sigma-Aldrich) (Buchs, Switzerland); Taxifolin (T) was from Biochemika (Fluka); Oleuropein (OG) was from Extrasynthèse (Genay France).

The stock solutions were prepared (under the conditions described by Bendini et al., 2003) by dissolving about $1 \mathrm{mg}$ of each standard in $1 \mathrm{~mL}$ of HPLC-grade methanol. The quantitative evaluation of individual phenolic compounds was performed using the five points curve (from 0.05 to $0.001 \mathrm{mg} /$ $\mathrm{ml}$ ) through the use of authentic standards.

\subsection{Chromatographic analysis of the standard phenolic mixture and the virgin olive oil's phenolic fraction}

HPLC analysis was carried out following the method described by Bendini et al. (2003). An HP 1100 Series (Hewlett Packard) equipped with a quaternary pump G1311A, a UV-Vis detector G1314A and, $a_{18}$ NUCLEODUR 100 column $5 \mu \mathrm{m}$ particle size, $25 \mathrm{~cm} \times 4.6 \mathrm{~mm}$ I.D (Macherey -Nagel: USA, Batch 34504032) was used. All solvents were filtered through a $0.45 \mu \mathrm{m}$ filter disk. A gradient elution was carried out using the following solvent system: mobile phase A: pure water-acetic acid (98:2, v/v), mobile phase B: methanol- acetonitrile $(1: 1, v / v)$. The linear gradient elution system was: from 0 to $25 \mathrm{~min}, 95 \%$ to $70 \% \mathrm{~A}$; from 25 to $35 \mathrm{~min}$, 70 to $60 \% \mathrm{~A}$; from 35 to $40 \mathrm{~min}, 60$ to $52 \% \mathrm{~A}$; from 40 to $50 \mathrm{~min}, 52$ to $30 \% \mathrm{~A}$; from 50 to $55 \mathrm{~min}, 30$ to $0 \% \mathrm{~A}$; from 55 to $65 \mathrm{~min}, 0$ to $95 \% \mathrm{~A}$; from 65 to $70 \mathrm{~min}, 95 \% \mathrm{~A}$, as post-run. All solvents used were of HPLC grade. The flow-rate was $0.5 \mathrm{~mL} \mathrm{~min}^{-1}$.

The chromatograms were recorded at 280 and $290 \mathrm{~nm}$. The injection volume was $20 \mu \mathrm{L}$. All the analyses were carried out at room temperature.

\subsection{Statistical analysis}

All analyses were carried out in triplicate and the results are reported as mean values. The data were treated statistically by means of the software Statistica version 6, for the description of factor of unification or discrimination, using the simple variance analysis (ANOVA) and the Test of NewmanKeuls. The correlation between the various analytical parameters is treated by the principal component analysis (PCA) and the ascending hierarchical clustering $(\mathrm{AHC})$ with the aim of highlighting the concept of biochemical markers for the structuring, identification and characterization of the various varieties.

On the whole of the quantitative variables significant differences were determined between the individuals by the influence of the medium (climate, Crop year ...) and of the varieties on these quantitative variables: Variety effect and year effect.

The effectiveness of the AHC depends primarily on the choice of the distance used. Our choice was related to the Euclidian distance to describe the similarity between the varieties. Knowing that the APC and the AHC are complementary, we are generally brought to use them jointly in order to analyze each problem.

\section{RESULTS AND DISCUSSION}

\subsection{Free acidity, peroxide value and UV spectrophotometric indices}

The parameters considered in this work (Table 1) of all the olive oils studied were within the estimated limits of EEC Reg. 2568/91 (1991) and therefore the oils could be classified into the category of extra virgin olive oil.

The variety significantly affects $(P \leq 0.01)$ free acidity, peroxide value and UV spectrophotometric indices (at 232 and $270 \mathrm{~nm}$ ), whereas no significant effect $(P \geq 0.05)$ regarding the year (harvest) was found with the exception of the indication at $270 \mathrm{~nm}$ where the effect was significant $(P \leq 0.05)$ (Table 2).

\subsection{Total phenols and $\mathbf{O}$-diphenols}

Total phenol contributes to the oxidative stability of olive oil to a large extent (Beltran et al., 2000; Gutiérrez et al., 2001; Baccouri et al., 2008). In our study this characterization sign was not influenced by the year $(P \geq 0.05)$ but the effect of variety effect was clearly underlined $(P \leq 00.001)$ (Table 2$)$.

The total phenol contents in the oil in all varieties were moderate (table 3 ). The phenol contents varied from $109.45 \pm 0.07$ to $322.18 \pm 0.04 \mathrm{mg} \mathrm{Kg}^{-1}$. This variation could be explained by the fact that the samples were collected at full maturity, when the skin was black and the fruit removal force was low.

Bonoli et al. (2004) and Beltrán et al. (2005) noted that oil from the last olive fruit maturation presented a lower level of phenolic compounds.

Our results were in good agreement with those obtained by many authors Dugo et al. (2004) in Sicily with the varietes 'Biancolilla', 'Nocellara del Belice', 'Cerasuola', 'Tonda Iblea' and 'Crastu', Ollivier et al. (2004) using the 'Bouteillon' and 'Salonenque' varieties, Tamendjari et al. (2009a, $2009 b, 2011$ ) with the local varieties Chemlal and Azeradj, Boukachabine et al. (2007) in Morocco with the Picholine variety.

Our varieties were categorized among the Greco, Slovak and Italian varieties according to a recent work carried out by the COI registered laboratory (COI, 2009).

With regards to the total phenols, the $O$-diphenols contribute effectively to the oxidative stability of olive oil. The obtained values varied between $52.9 \pm 0.14$ and $95.03 \pm 0.04 \mathrm{mg} \mathrm{Kg}^{-1}$ (table 2).

Our results were similar to those obtained by Baccouri et al. (2008) with local Tunisian varieties. The variance analysis showed a high significant difference $(P \leq 0.001)$ concerning the variety effect and no significant effect for year $(P \geq 0.05)$. 
Table 1

Analytical characteristics of Algerian extra virgin olive oils at different years of harvest.

\begin{tabular}{|c|c|c|c|c|c|c|}
\hline \multicolumn{2}{|c|}{$\begin{array}{c}\text { Population Variety } \\
\text { Crop year }\end{array}$} & \multirow{2}{*}{$\begin{array}{c}\text { Free Acidity } \\
\text { (\%) }\end{array}$} & \multirow{2}{*}{$\begin{array}{c}\begin{array}{c}\text { Peroxide } \\
\text { value* }^{*}\end{array} \\
10.8 \pm 0.14\end{array}$} & \multirow{2}{*}{$\begin{array}{c}\text { K232 } \\
2.28 \pm 0.02\end{array}$} & \multirow{2}{*}{$\begin{array}{c}\text { K270 } \\
0.23 \pm 0.01\end{array}$} & \multirow{2}{*}{$\begin{array}{c}\begin{array}{c}\text { Size of } \\
\text { olive }\end{array} \\
\text { Great }\end{array}$} \\
\hline Azeradj & Year 1 & & & & & \\
\hline & Year 2 & $0.57 \pm 0.01$ & $10.0 \pm 0.00$ & $1.16 \pm 0.05$ & $0.21 \pm 0.01$ & \\
\hline \multirow[t]{2}{*}{ Chemlal } & Year 1 & $0.23 \pm 0.01$ & $15.05 \pm 0.07$ & $2.38 \pm 0.01$ & $0.21 \pm 0.01$ & Small \\
\hline & Year 2 & $0.34 \pm 0.01$ & $13.85 \pm 0.07$ & $2.12 \pm 0.02$ & $0.26 \pm 0.01$ & \\
\hline \multirow[t]{2}{*}{ Limli } & Year 1 & $0.37 \pm 0.01$ & $15.64 \pm 0.28$ & $2.48 \pm 0.01$ & $0.21 \pm 0.01$ & Small \\
\hline & Year 2 & $0.47 \pm 0.01$ & $13.30 \pm 0.13$ & $2.32 \pm 0.01$ & $0.29 \pm 0.01$ & \\
\hline \multirow[t]{2}{*}{ Aimell } & Year 1 & $0.28 \pm 0.01$ & $11.1 \pm 0.00$ & $2.21 \pm 0.00$ & $0.22 \pm 0.00$ & Small \\
\hline & Year 2 & $0.39 \pm 0.01$ & $6.08 \pm 0.11$ & $1.94 \pm 0.01$ & $0.19 \pm 0.01$ & \\
\hline \multirow[t]{2}{*}{ Aghenfas } & Year 1 & $0.41 \pm 0.01$ & $16.00 \pm 0.01$ & $2.92 \pm 0.01$ & $0.25 \pm 0.01$ & Great \\
\hline & Year 2 & $0.51 \pm 0.01$ & $18.1 \pm 0.14$ & $2.43 \pm 0.06$ & $0.25 \pm 0.00$ & \\
\hline \multirow[t]{2}{*}{ Grosse du Hamma } & Year 1 & $0.40 \pm 0.01$ & $17.88 \pm 0.08$ & $2.36 \pm 0.01$ & $0.13 \pm 0.01$ & Great \\
\hline & Year 2 & $0.30 \pm 0.01$ & $20.70 \pm 0.13$ & $3.02 \pm 0.01$ & $0.37 \pm 0.01$ & \\
\hline \multirow[t]{2}{*}{ Aghenaou } & Year 1 & $0.40 \pm 0.01$ & $15.5 \pm 0.42$ & $2.54 \pm 0.01$ & $0.20 \pm 0.00$ & Great \\
\hline & Year 2 & $0.30 \pm 0.01$ & $12.50 \pm 0.35$ & $1.96 \pm 0.01$ & $0.18 \pm 0.00$ & \\
\hline \multirow[t]{2}{*}{ Hamra } & Year 1 & $0.29 \pm 0.01$ & $10.6 \pm 0.14$ & $1.85 \pm 0.01$ & $0.23 \pm 0.01$ & Small \\
\hline & Year 2 & $0.3 \pm 0.0$ & $6.2 \pm 0.0$ & $1.79 \pm 0.01$ & $0.21 \pm 0.01$ & \\
\hline \multirow[t]{2}{*}{ Boughenfas } & Year 1 & $0.20 \pm 0.01$ & $10.55 \pm 0.07$ & $1.87 \pm 0.03$ & $0.22 \pm 0.00$ & Small \\
\hline & Year 2 & $0.13 \pm 0.01$ & $11.45 \pm 0.06$ & $2.35 \pm 0.01$ & $0.28 \pm 0.00$ & \\
\hline \multirow[t]{2}{*}{ X-Aghenfas } & Year 1 & $0.35 \pm 0.01$ & $11.4 \pm 0.14$ & $2.49 \pm 0.01$ & $0.27 \pm 0.01$ & Great \\
\hline & Year 2 & $0.29 \pm 0.01$ & $16.67 \pm 0.45$ & $2.88 \pm 0.01$ & $0.22 \pm 0.01$ & \\
\hline \multirow[t]{2}{*}{ Aberkane } & Year 1 & $0.32 \pm 0.01$ & $12.52 \pm 0.04$ & $2.86 \pm 0.07$ & $0.28 \pm 0.00$ & Great \\
\hline & Year 2 & $0.42 \pm 0.01$ & $14.55 \pm 0.07$ & $1.14 \pm 0.02$ & $0.21 \pm 0.01$ & \\
\hline \multirow[t]{2}{*}{ Neb djemel } & Year 1 & $0.41 \pm 0.01$ & $12.10 \pm 0.13$ & $2.21 \pm 0.01$ & $0.13 \pm 0.00$ & Middle \\
\hline & Year 2 & $0.25 \pm 0.00$ & $12.57 \pm 0.09$ & $1.78 \pm 0.02$ & $0.18 \pm 0.01$ & \\
\hline \multirow[t]{2}{*}{ Mekki } & Year 1 & $0.38 \pm 0.01$ & $14.2 \pm 0.42$ & $2.65 \pm 0.05$ & $0.33 \pm 0.01$ & Small \\
\hline & Year 2 & $0.30 \pm 0.01$ & $20.45 \pm 0.08$ & $2.33 \pm 0.01$ & $0.25 \pm 0.01$ & \\
\hline \multirow{2}{*}{$\begin{array}{l}\text { Bouchouk de } \\
\text { Guergour }\end{array}$} & Year 1 & $0.44 \pm 0.01$ & $12.09 \pm 0.28$ & $3.07 \pm 0.01$ & $0.2 \pm 0.00$ & Great \\
\hline & Year 2 & $0.23 \pm 0.01$ & $6.69 \pm 0.14$ & $2.02 \pm 0.01$ & $0.23 \pm 0.01$ & \\
\hline \multirow[t]{2}{*}{ Bouichret } & Year 1 & $0.41 \pm 0.01$ & $19.25 \pm 0.07$ & $1.69 \pm 0.01$ & $0.18 \pm 0.01$ & Middle \\
\hline & Year 2 & $0.22 \pm 0.01$ & $15.84 \pm 0.07$ & $2.00 \pm 0.0$ & $0.20 \pm 0.00$ & \\
\hline \multirow[t]{2}{*}{ Rougette de la mitidja } & Year 1 & $0.43 \pm 0.01$ & $12.45 \pm 0.49$ & $2.32 \pm 0.01$ & $0.21 \pm 0.00$ & Middle \\
\hline & Year 2 & $0.52 \pm 0.01$ & $20.6 \pm 0.28$ & $3.32 \pm 0.01$ & $0.23 \pm 0.01$ & \\
\hline \multirow[t]{2}{*}{ Rougette de Guelma } & Year 1 & $0.38 \pm 0.01$ & $15.48 \pm 0.01$ & $2.27 \pm 0.01$ & $0.23 \pm 0.01$ & Great \\
\hline & Year 2 & $0.27 \pm 0.01$ & $16.59 \pm 0.14$ & $2.35 \pm 0.01$ & $0.24 \pm 0.01$ & \\
\hline \multirow[t]{2}{*}{ Bounguerguerb } & Year 1 & $0.33 \pm 0.01$ & $11.47 \pm 0.13$ & $2.09 \pm 0.07$ & $0.20 \pm 0.01$ & Middle \\
\hline & Year 2 & $0.51 \pm 0.01$ & $17.89 \pm 0.14$ & $2.02 \pm 0.01$ & $0.29 \pm 0.01$ & \\
\hline \multirow[t]{2}{*}{ Ronde de miliana } & Year 1 & $0.39 \pm 0.01$ & $12.72 \pm 0.04$ & $2.39 \pm 0.02$ & $0.17 \pm 0.00$ & Middle \\
\hline & Year 2 & $0.25 \pm 0.01$ & $23.95 \pm 0.07$ & $3.22 \pm 0.01$ & $0.24 \pm 0.01$ & \\
\hline Sigoise & Year 1 & $0.33 \pm 0.01$ & $10.94 \pm 0.07$ & $2.37 \pm 0.02$ & $0.2 \pm 0.00$ & Great \\
\hline & Year 2 & $0.42 \pm 0.01$ & $14.90 \pm 0.01$ & $2.50 \pm 0.01$ & $0.25 \pm 0.01$ & \\
\hline Blanquette de Guelma & Year 1 & $0.34 \pm 0.01$ & $12.75 \pm 0.35$ & $2.36 \pm 0.02$ & $0.22 \pm 0.01$ & Middle \\
\hline & Year 2 & $0.36 \pm 0.00$ & $17.10 \pm 0.01$ & $2.14 \pm 0.01$ & $0.24 \pm 0.01$ & \\
\hline
\end{tabular}

${ }^{*}$ meq $\mathrm{O}_{2} \mathrm{Kg}^{-1}$ 
Table 2

Variance analysis ANOVA: Analytical characteristics

\begin{tabular}{lcccccc}
\hline & Free acidity & $\begin{array}{c}\text { Peroxide } \\
\text { value }\end{array}$ & K232 & K270 & $\begin{array}{c}\text { Total } \\
\text { phenols }\end{array}$ & o-diphenols \\
\cline { 2 - 7 } & $\mathbf{F}(\mathbf{p}) \mathbf{D D L}$ & $\mathbf{F}(\mathbf{p}) \mathbf{D D L}$ & $\mathbf{F}(\mathbf{p}) \mathbf{D D L}$ & $\mathbf{F}(\mathbf{p}) \mathbf{D D L}$ & $\mathbf{F}(\mathbf{p}) \mathbf{D D L}$ & $\mathbf{F}(\mathbf{p}) \mathbf{D D L}$ \\
\hline Population variety & 4.43820 & 5.50120 & 3.04620 & 1.81920 & 14.85620 & 10.34320 \\
Effect & $(p<0.001)$ & $(p \leq 0.001)$ & $(p<0.001)$ & $(p \leq 0.001)$ & $(p<0.001)$ & $(p \leq 0.001)$ \\
Year Effect & 0.1221 & 2.7871 & 1.8711 & 5.6841 & 0.0521 & 1.6701 \\
& $(p>0.05)$ & $(p>0.05)$ & $(p>0.05)$ & $(p>0.05)$ & $(p>0.05)$ & $(p>0.05)$ \\
\hline
\end{tabular}

Significance level at $P \leq 0.001 \mathrm{HS} ; \mathrm{P} \leq 0.01 \mathrm{MS} ; \mathrm{P} \leq 0.05 \mathrm{~S} ; \mathrm{P} \geq 0.05 \mathrm{~N}$

\subsection{HPLC analysis of phenolic compounds}

The results obtained showed that the variety population did not contain gallic acid in its profile (table 4).

The variance analysis showed a high significant effect for variety $(P \leq 0.001)$ and no significant difference for the year effect $(P \geq 0.05)$ for all the individual phenol compounds except for ferulic acid where the year effect was moderately significant $(P \leq 0.01)$ (table 5).

The phenol compound contents in all the oil was moderate and were in the same range as that reported by Dugo et al. (2004) in Italy with the varieties 'Biancolilla', 'Nocellara del Belice', 'Cerasuola', 'Tonda Iblea' and 'Crastu'; Tsimidou et al. (1992) with Greece's five local varieties; OliverasLópez et al. (2007) with Spanish and Italian varieties (Picual, Hojiblanca, Arbequina, Picuda, Taggiasca).

A similarity was found between our results and those reported of Veillet et al. (2009) for vanilic acid and $p$-coumaric acid for some of the varieties.

It appears from the data in Table 4 that tyrosol, hydroxytyrosol and 4-hydroxybenzoic acid (4-HBA), were the principal phenol compounds identified in all varieties: the values ranged respectively from $9.02 \pm 0.01$ to $36.30 \pm 0.01 \mathrm{mg} \mathrm{Kg}^{-1}$; $1.06 \pm 0.01$ to $14.92 \pm 0.01 \mathrm{mg} \mathrm{Kg}^{-1}$ and $1.01 \pm 0.01$ to $11.97 \pm 0.01 \mathrm{mg} \mathrm{Kg}^{-1}$.

As for caffeic acid $(0.33 \pm 0.01$ to $4.74 \pm 0.01 \mathrm{mg}$ $\mathrm{Kg}^{-1}$ ), 3-methoxybenzoic acid (0.00 to $12.19 \pm$ $\pm 0.01 \mathrm{mg} \mathrm{Kg}^{-1}$ ) and oleuropein (0.0 to $13.07 \pm$ $\pm 0.01 \mathrm{mg} \mathrm{Kg}^{-1}$ ) were moderately represented and the rest was weakly represented. Forcadell et al. (1987); Montedoro et al. (1992); Akasbi et al. (1993); Litridou et al. (1997); Artajo et al. (2007) have identified the same majority phenol compound.

Visioli et al. (2002) reported that the more dominant phenol compounds in olive oil that contribute to its efficacy for stability and taste were hydroxytyrosol, tyrosol and oleuropein.

The varieties of large fruits (Grosse $d u$ Hamma, Aghenfas, Azeradj, Aguenaou, Aberkane, Bouchouk de Guergour, X-Aghenfas, Rougette de Guelma and Sigoise) were clearly particular and were characterized with higher levels of the three majority individual phenolic compounds (tyrosol, hydroxytyrosol and 4-hydroxybenzoic acid.
Otherwise the varieties with small fruits (Hamra, Chemlal, Boughenfas, Limli, Aimel and Mekki) have presented the highest levels of oleuropein. This result was in agreement with that reported by Amiot et al. (1986) and Ryan et al. (1998).

\subsection{Multi dimensional analysis}

At the threshold of $5 \%$, the correlation between variables was significant. By using the coefficient of Pearson, we observed positive correlations between variables, notably the quantitative variables in the variety Mekki with 3-methoxybenzoic acid, Chemlal with p-coumaric acid; Hamra with oleuropein; Boughenfes with protocatechuic acid; Bounguergueb with: o-coumaric acid, taxifolin and cinnamic acid; Grosse du Hamma with hyroxytyrosol.

p-coumaric acid was strongly correlated with ferulic acid, o-coumaric acid with cinnamic acid, taxifolin with o-coumaric and cinnamic acids. A correlation was also noted between vanillic acid and oleuropein. However, certain variables were weakly correlated.

The correlation circle (Fig. 1) corresponds to the projection of the initial variables on a plan with two dimensions constituted by two unique factors (F1 and F2) and account for $32 \%$. This projection of points on the circle showed a middle dispersion of the variables on the two axis, which means that the varieties studied present a great diversity from the point of view of physico-chemical and biochemical aspects.

The projection was relatively far from the center for some variables, notably vanillic acid, oleuropein, protocatechuic acid and $p$-coumaric acid, and the peroxide index which was correlated to $\mathrm{F} 1$. This explains its strong dependence to this axis, but axis 2 is clearly linked for cinnamic acid, o-coumaric acid, total phenol compounds, tyrosol, protocatechuic acid, ferulic acid and the 3-methoxybenzoic acid.

The study of the contribution of each variable allowed for prioritizing the responsible characters of each axis formation. We noticed that most of the variables contributed very little to an explanation of each axis, except for some variables such as protocatechuic acid, $p$-coumaric acid, ferulic acid and oleuropein, which are reflected in axis 1 . Vanillic acid, Taxifolin, o-coumaric acid and cinnamic acid 
Table 3

Average value of total phenols (TP) and o-diphenols (o-diph) ( $\mathrm{mg} \mathrm{kg}^{-1}$ as gallic acid) of the phenolic fraction determined by spectrophotometry of the virgin olive oils of several varieties at different years of harvest

\begin{tabular}{|c|c|c|c|}
\hline \multicolumn{2}{|c|}{$\begin{array}{c}\text { Population Variety } \\
\text { Crop year }\end{array}$} & \multirow{2}{*}{$\begin{array}{l}\text { Total phenols } \\
217.68 \pm 0.11\end{array}$} & \multirow{2}{*}{$\begin{array}{l}\text { o-diphenols } \\
91.00 \pm 0.00\end{array}$} \\
\hline Azeradj & Year 1 & & \\
\hline & Year 2 & $270.11 \pm 0.16$ & $77.26 \pm 0.01$ \\
\hline \multirow[t]{2}{*}{ Chemlal } & Year 1 & $233.05 \pm 0.07$ & $73.05 \pm 0.07$ \\
\hline & Year 2 & $235.78 \pm 0.04$ & $87.04 \pm 0.06$ \\
\hline \multirow[t]{2}{*}{ Limli } & Year 1 & $125.95 \pm 0.08$ & $64.53 \pm 0.04$ \\
\hline & Year 2 & $128.55 \pm 0.1$ & $52.90 \pm 0.14$ \\
\hline \multirow[t]{2}{*}{ Aimell } & Year 1 & $143.8 \pm 0.07$ & $60.5 \pm 0.71$ \\
\hline & Year 2 & $185.53 \pm 0.10$ & $53.9 \pm 0.14$ \\
\hline \multirow[t]{2}{*}{ Aghenfas } & Year 1 & $210.33 \pm 0.04$ & $95.03 \pm 0.04$ \\
\hline & Year 2 & $228.69 \pm 0.02$ & $80.8 \pm 0.28$ \\
\hline \multirow[t]{2}{*}{ Grosse du Hamma } & Year 1 & $285.12 \pm 0.02$ & $90.25 \pm 0.35$ \\
\hline & Year 2 & $322.18 \pm 0.04$ & $85.15 \pm 0.21$ \\
\hline \multirow[t]{2}{*}{ Aghenaou } & Year 1 & $203.27 \pm 0.09$ & $64.15 \pm 0.21$ \\
\hline & Year 2 & $191.5 \pm 0.71$ & $86.25 \pm 0.35$ \\
\hline \multirow[t]{2}{*}{ Hamra } & Year 1 & $116.76 \pm 0.01$ & $64.23 \pm 0.04$ \\
\hline & Year 2 & $166 \pm 0.00$ & $53.29 \pm 0.08$ \\
\hline \multirow[t]{2}{*}{ Boughenfas } & Year 1 & $299.45 \pm 0.07$ & $62.25 \pm 0.35$ \\
\hline & Year 2 & $167.63 \pm 0.04$ & $58.85 \pm 0.21$ \\
\hline \multirow[t]{2}{*}{ X-Aghenfas } & Year 1 & $232.58 \pm 0.01$ & $75.50 \pm 0.71$ \\
\hline & Year 2 & $185.48 \pm 0.03$ & $80.15 \pm 0.21$ \\
\hline \multirow[t]{2}{*}{ Aberkane } & Year 1 & $150.17 \pm 0.08$ & $63.9 \pm 0.14$ \\
\hline & Year 2 & $137.18 \pm 0.22$ & $55.05 \pm 0.07$ \\
\hline \multirow[t]{2}{*}{ Neb djemel } & Year 1 & $196.78 \pm 0.03$ & $79.05 \pm 0.07$ \\
\hline & Year 2 & $194.00 \pm 0.00$ & $55.04 \pm 0.05$ \\
\hline \multirow[t]{2}{*}{ Mekki } & Year 1 & $195.49 \pm 0.01$ & $56.82 \pm 0.02$ \\
\hline & Year 2 & $122.29 \pm 0.02$ & $70.04 \pm 0.06$ \\
\hline \multirow[t]{2}{*}{ Bouchouk de Guergour } & Year 1 & $122.35 \pm 0.01$ & $68.5 \pm 0.71$ \\
\hline & Year 2 & $199.39 \pm 0.01$ & $61.13 \pm 0.18$ \\
\hline \multirow[t]{2}{*}{ Bouichret } & Year 1 & $201.6 \pm 0.00$ & $66.16 \pm 0.06$ \\
\hline & Year 2 & $194.11 \pm 0.06$ & $58.18 \pm 0.04$ \\
\hline \multirow[t]{2}{*}{ Rougette de la mitidja } & Year 1 & $109.45 \pm 0.07$ & $75.05 \pm 0.07$ \\
\hline & Year 2 & $113.4 \pm 0.14$ & $68.5 \pm 0.71$ \\
\hline \multirow[t]{2}{*}{ Rougette de Guelma } & Year 1 & $122.37 \pm 0.04$ & $68.04 \pm 0.05$ \\
\hline & Year 2 & $119.58 \pm 0.04$ & $74.25 \pm 0.35$ \\
\hline \multirow[t]{2}{*}{ Bounguerguerb } & Year 1 & $149.35 \pm 0.01$ & $84.5 \pm 0.71$ \\
\hline & Year 2 & $145.38 \pm 0.01$ & $80 \pm 0.00$ \\
\hline \multirow[t]{2}{*}{ Ronde de miliana } & Year 1 & $217.63 \pm 0.04$ & $91.25 \pm 0.35$ \\
\hline & Year 2 & $253.09 \pm 0.02$ & $77.12 \pm 0.03$ \\
\hline \multirow[t]{2}{*}{ Sigoise } & Year 1 & $263.15 \pm 0.07$ & $73.55 \pm 0.01$ \\
\hline & Year 2 & $234.25 \pm 0.01$ & $87.25 \pm 0.35$ \\
\hline \multirow[t]{2}{*}{ Blanquette de Guelma } & Year 1 & $275.90 \pm 0.01$ & $60.25 \pm 0.35$ \\
\hline & Year 2 & $218.89 \pm 0.02$ & $52.9 \pm 0.14$ \\
\hline
\end{tabular}


Table 4

Phenolic compound composition $\left(\mathrm{mg} \mathrm{kg}^{-1}\right)$ in different Algerian mono-cultivar extra virgin olive oils at different years of harvest

\begin{tabular}{|c|c|c|c|c|c|c|c|c|c|c|c|c|c|}
\hline $\begin{array}{c}\text { Phenolic } \\
\text { compounds } \\
\text { Population Variety }\end{array}$ & $\begin{array}{l}\text { Proto- } \\
\text { catechic } \\
\text { acid }\end{array}$ & $\begin{array}{l}\text { Hydroxy- } \\
\text { tyrosol }\end{array}$ & Tyrosol & $\begin{array}{l}\text { 4-hydroxy- } \\
\text { benzoic acid }\end{array}$ & $\begin{array}{l}\text { Caffeic } \\
\text { acid }\end{array}$ & $\begin{array}{l}\text { Vanillic } \\
\text { acid }\end{array}$ & $\begin{array}{c}\text { p- } \\
\text { coumaric } \\
\text { acid }\end{array}$ & Taxifolin & $\begin{array}{l}\text { Ferulic } \\
\text { acid }\end{array}$ & $\begin{array}{l}\text { o-coumaric } \\
\text { acid }\end{array}$ & Oleuropein & $\begin{array}{c}\text { 3-methox } \\
\text { benzoic acid }\end{array}$ & $\begin{array}{l}\text { Cinnamic } \\
\text { acid }\end{array}$ \\
\hline Azeradj 1 & $0.0 \pm 0.0$ & $2.84 \pm 0.01$ & $26.28 \pm 0.07$ & $7.37 \pm 0.08$ & $4.74 \pm 0.01$ & $0.81 \pm 0.01$ & $0.0 \pm 0.0$ & $1.12 \pm 0.01$ & $0.40 \pm 0.01$ & $0.07 \pm 0.0$ & $1.21 \pm 0.01$ & $0.0 \pm 0.0$ & $0.02 \pm 0.0$ \\
\hline Azeradj 2 & $0.0 \pm 0.0$ & $3.00 \pm 0.00$ & $13.27 \pm 0.01$ & $11.36 \pm 0.01$ & $3.00 \pm 0.0$ & $0.66 \pm 0.01$ & $0.0 \pm 0.0$ & $0.75 \pm 0.01$ & $0.03 \pm 0.01$ & $0.11 \pm 0.01$ & $2.05 \pm 0.07$ & $0.0 \pm 0.0$ & $0.11 \pm 0.0$ \\
\hline Mekki 1 & $0.0 \pm 0.0$ & $1.06 \pm 0.01$ & $11.16 \pm 0.01$ & $4.05 \pm 0.01$ & $0.72 \pm 0.01$ & $0.39 \pm 0.01$ & $0.0 \pm 0.0$ & $0.75 \pm 0.07$ & $0.10 \pm 0.0$ & $0.00 \pm 0.0$ & $0.00 \pm 0.0$ & $12.19 \pm 0.01$ & $0.0 \pm 0.0$ \\
\hline Mekki 2 & $0.0 \pm 0.0$ & $1.43 \pm 0.01$ & $9.02 \pm 0.01$ & $4.47 \pm 0.01$ & $1.16 \pm 0.01$ & $0.33 \pm 0.01$ & $0.0 \pm 0.0$ & $0.27 \pm 0.01$ & $0.03 \pm 0.01$ & $0.00 \pm 0.0$ & $0.00 \pm 0.0$ & $2.05 \pm 0.07$ & $0.0 \pm 0.0$ \\
\hline Neb djemel 1 & $0.0 \pm 0.0$ & $7.27 \pm 0.01$ & $15.15 \pm 0.01$ & $6.08 \pm 0.01$ & $2.00 \pm 0.0$ & $0.16 \pm 0.01$ & $0.0 \pm 0.0$ & $0.47 \pm 0.01$ & $0.0 \pm 0.0$ & $0.04 \pm 0.01$ & $0.00 \pm 0.0$ & $1.38 \pm 0.01$ & $0.03 \pm 0.01$ \\
\hline Neb djemel 2 & $0.0 \pm 0.0$ & $4.44 \pm 0.01$ & $17.14 \pm 0.01$ & $3.25 \pm 0.01$ & $1.56 \pm 0.01$ & $0.26 \pm 0.01$ & $0.0 \pm 0.0$ & $0.34 \pm 0.01$ & $0.0 \pm 0.0$ & $0.09 \pm 0.01$ & $0.00 \pm 0.0$ & $1.05 \pm 0.05$ & $0.04 \pm 0.01$ \\
\hline Chemlal 1 & $4.03 \pm 0.04$ & $3.28 \pm 0.01$ & $13.60 \pm 0.01$ & $1.92 \pm 0.01$ & $1.65 \pm 0.07$ & $1.55 \pm 0.07$ & $1.92 \pm 0.01$ & $0.36 \pm 0.01$ & $0.75 \pm 0.07$ & $0.02 \pm 0.01$ & $4.36 \pm 0.01$ & $0.0 \pm 0.0$ & $0.0 \pm 0.0$ \\
\hline Chemlal 2 & $0.0 \pm 0.0$ & $4.21 \pm 0.01$ & $19.16 \pm 0.01$ & $4.66 \pm 0.01$ & $2.25 \pm 0.01$ & $0.56 \pm 0.01$ & $0.11 \pm 0.01$ & $1.43 \pm 0.01$ & $0.0 \pm 0.0$ & $0.12 \pm 0.01$ & $0.00 \pm 0.0$ & $2.31 \pm 0.01$ & $0.05 \pm 0.01$ \\
\hline Hamra 1 & $0.0 \pm 0.0$ & $1.14 \pm 0.01$ & $10.03 \pm 0.04$ & $4.05 \pm 0.07$ & $0.33 \pm 0.01$ & $0.65 \pm 0.01$ & $0.21 \pm 0.01$ & $0.46 \pm 0.01$ & $0.55 \pm 0.07$ & $0.00 \pm 0.0$ & $8.24 \pm 0.01$ & $0.42 \pm 0.03$ & $0.1 \pm 0.0$ \\
\hline Hamra 2 & $0.0 \pm 0.0$ & $4.11 \pm 0.01$ & $20.69 \pm 0.01$ & $4.90 \pm 0.01$ & $2.35 \pm 0.01$ & $1.67 \pm 0.01$ & $0.21 \pm 0.01$ & $0.24 \pm 0.01$ & $0.47 \pm 0.01$ & $0.53 \pm 0.01$ & $13.07 \pm 0.01$ & $2.58 \pm 0.01$ & $0.24 \pm 0.01$ \\
\hline $\begin{array}{l}\text { Blanquette } \\
\text { de Guelma } 1\end{array}$ & $0.0 \pm 0.0$ & $4.76 \pm 0.01$ & $19.52 \pm 0.01$ & $5.79 \pm 0.06$ & $2.31 \pm 0.01$ & $0.67 \pm 0.01$ & $0.0 \pm 0.0$ & $0.99 \pm 0.01$ & $0.13 \pm 0.01$ & $0.23 \pm 0.01$ & $2.07 \pm 0.09$ & $1.14 \pm 0.01$ & $0.09 \pm 0.01$ \\
\hline $\begin{array}{l}\text { Blanquette } \\
\text { de Guelma } 2\end{array}$ & $0.0 \pm 0.0$ & $8.96 \pm 0.01$ & $21.55 \pm 0.07$ & $4.24 \pm 0.01$ & $3.96 \pm 0.01$ & $0.56 \pm 0.01$ & $0.0 \pm 0.0$ & $1.42 \pm 0.01$ & $0.26 \pm 0.01$ & $0.57 \pm 0.01$ & $1.35 \pm 0.07$ & $0.76 \pm 0.01$ & $0.05 \pm 0.01$ \\
\hline Limli 1 & $0.0 \pm 0.0$ & $3.14 \pm 0.01$ & $13.09 \pm 0.01$ & $6.23 \pm 0.01$ & $1.35 \pm 0.01$ & $0.18 \pm 0.01$ & $0.0 \pm 0.0$ & $0.32 \pm 0.01$ & $0.09 \pm 0.0$ & $0.0 \pm 0.0$ & $0.00 \pm 0.0$ & $2.05 \pm 0.07$ & $0.0 \pm 0.0$ \\
\hline Limli 2 & $0.0 \pm 0.0$ & $2.89 \pm 0.01$ & $18.19 \pm 0.01$ & $9.21 \pm 0.01$ & $2.56 \pm 0.01$ & $0.21 \pm 0.01$ & $0.0 \pm 0.0$ & $0.53 \pm 0.01$ & $0.05 \pm 0.01$ & $0.0 \pm 0.0$ & $0.00 \pm 0.0$ & $1.54 \pm 0.01$ & $0.0 \pm 0.0$ \\
\hline Aberkane1 & $0.0 \pm 0.0$ & $1.10 \pm 0.01$ & $16.69 \pm 0.01$ & $11.91 \pm 0.01$ & $0.42 \pm 0.01$ & $0.19 \pm 0.01$ & $0.0 \pm 0.0$ & $0.46 \pm 0.01$ & $0.45 \pm 0.01$ & $0.06 \pm 0.01$ & $0.00 \pm 0.0$ & $0.00 \pm 0.0$ & $0.07 \pm 0.01$ \\
\hline Aberkane 2 & $0.0 \pm 0.0$ & $1.22 \pm 0.01$ & $18.45 \pm 0.01$ & $9.39 \pm 0.01$ & $1.3 \pm 0.14$ & $0.37 \pm 0.01$ & $0.0 \pm 0.0$ & $0.90 \pm 0.01$ & $0.08 \pm 0.01$ & $0.18 \pm 0.01$ & $0.00 \pm 0.0$ & $0.00 \pm 0.0$ & $0.04 \pm 0.01$ \\
\hline Aimell 1 & $2.02 \pm 0.0$ & $2.23 \pm 0.01$ & $18.05 \pm 0.01$ & $1.19 \pm 0.01$ & $0.84 \pm 0.01$ & $0.30 \pm 0.01$ & $0.0 \pm 0.0$ & $0.32 \pm 0.01$ & $0.06 \pm 0.01$ & $0.05 \pm 0.01$ & $0.00 \pm 0.0$ & $0.29 \pm 0.01$ & $0.0 \pm 0.0$ \\
\hline Aimell 2 & $1.33 \pm 0.01$ & $6.31 \pm 0.01$ & $18.30 \pm 0.01$ & $5.91 \pm 0.01$ & $2.08 \pm 0.01$ & $0.32 \pm 0.01$ & $0.0 \pm 0.0$ & $0.75 \pm 0.07$ & $0.14 \pm 0.01$ & $0.03 \pm 0.01$ & $0.00 \pm 0.0$ & $1.02 \pm 0.01$ & $0.0 \pm 0.0$ \\
\hline $\begin{array}{l}\text { Rougettle } \\
\text { de la mitidja } 1\end{array}$ & $0.0 \pm 0.0$ & $4.10 \pm 0.01$ & $14.56 \pm 0.01$ & $1.01 \pm 0.01$ & $0.94 \pm 0.01$ & $0.22 \pm 0.01$ & $0.16 \pm 0.01$ & $0.56 \pm 0.01$ & $0.03 \pm 0.00$ & $0.23 \pm 0.01$ & $1.45 \pm 0.01$ & $0.00 \pm 0.0$ & $0.09 \pm 0.0$ \\
\hline $\begin{array}{l}\text { Rougette } \\
\text { de la mitidja } 2\end{array}$ & $0.0 \pm 0.0$ & $4.56 \pm 0.01$ & $19.58 \pm 0.01$ & $4.20 \pm 0.01$ & $1.82 \pm 0.01$ & $0.21 \pm 0.01$ & $0.30 \pm 0.01$ & $0.66 \pm 0.01$ & $0.13 \pm 0.01$ & $0.29 \pm 0.01$ & $1.16 \pm 0.01$ & $2.15 \pm 0.07$ & $0.12 \pm 0.01$ \\
\hline Aghenaou 1 & $0.0 \pm 0.0$ & $1.66 \pm 0.01$ & $14.03 \pm 0.01$ & $3.34 \pm 0.01$ & $1.98 \pm 0.01$ & $0.26 \pm 0.01$ & $0.0 \pm 0.0$ & $0.11 \pm 0.01$ & $0.0 \pm 0.0$ & $0.00 \pm 0.0$ & $0.00 \pm 0.0$ & $1.07 \pm 0.01$ & $0.04 \pm 0.01$ \\
\hline Aghenaou 2 & $0.0 \pm 0.0$ & $2.39 \pm 0.01$ & $18.06 \pm 0.01$ & $4.79 \pm 0.01$ & $2.04 \pm 0.06$ & $0.37 \pm 0.01$ & $0.0 \pm 0.0$ & $0.28 \pm 0.01$ & $0.08 \pm 0.01$ & $0.00 \pm 0.0$ & $0.00 \pm 0.0$ & $2.02 \pm 0.02$ & $0.0 \pm 0.0$ \\
\hline Boughenfas 1 & $5.44 \pm 0.01$ & $2.56 \pm 0.01$ & $16.64 \pm 0.01$ & $7.66 \pm 0.01$ & $2.20 \pm 0.01$ & $0.53 \pm 0.01$ & $0.0 \pm 0.0$ & $0.57 \pm 0.01$ & $0.09 \pm 0.01$ & $0.07 \pm 0.01$ & $2.51 \pm 0.01$ & $2.69 \pm 0.01$ & $0.0 \pm 0.0$ \\
\hline Boughenfas 2 & $1.34 \pm 0.01$ & $2.77 \pm 0.01$ & $26.34 \pm 0.01$ & $11.37 \pm 0.01$ & $1.04 \pm 0.01$ & $0.20 \pm 0.01$ & $0.0 \pm 0.0$ & $0.93 \pm 0.01$ & $0.0 \pm 0.0$ & $0.08 \pm 0.01$ & $1.65 \pm 0.01$ & $3.76 \pm 0.01$ & $0.0 \pm 0.0$ \\
\hline Bouichret 1 & $0.0 \pm 0.0$ & $3.10 \pm 0.01$ & $14.55 \pm 0.01$ & $1.01 \pm 0.01$ & $0.97 \pm 0.01$ & $0.42 \pm 0.01$ & $0.22 \pm 0.01$ & $0.76 \pm 0.01$ & $0.07 \pm 0.01$ & $0.13 \pm 0.01$ & $0.00 \pm 0.0$ & $1.14 \pm 0.01$ & $0.21 \pm 0.01$ \\
\hline Bouichret 2 & $0.0 \pm 0.0$ & $4.16 \pm 0.01$ & $13.52 \pm 0.01$ & $3.20 \pm 0.01$ & $1.02 \pm 0.01$ & $0.21 \pm 0.01$ & $0.13 \pm 0.01$ & $0.46 \pm 0.01$ & $0.11 \pm 0.01$ & $0.19 \pm 0.01$ & $0.00 \pm 0.0$ & $2.05 \pm 0.07$ & $0.12 \pm 0.01$ \\
\hline Aghentas 1 & $0.0 \pm 0.0$ & $3.19 \pm 0.01$ & $18.36 \pm 0.01$ & $11.97 \pm 0.01$ & $0.84 \pm 0.01$ & $0.53 \pm 0.01$ & $0.0 \pm 0.0$ & $0.63 \pm 0.01$ & $0.02 \pm 0.01$ & $0.00 \pm 0.0$ & $5.14 \pm 0.01$ & $0.00 \pm 0.0$ & $0.02 \pm 0.00$ \\
\hline Aghentas 2 & $0.0 \pm 0.0$ & $3.91 \pm 0.01$ & $25.07 \pm 0.01$ & $6.26 \pm 0.01$ & $2.85 \pm 0.07$ & $0.33 \pm 0.01$ & $0.0 \pm 0.0$ & $0.47 \pm 0.01$ & $0.05 \pm 0.01$ & $0.00 \pm 0.0$ & $0.81 \pm 0.01$ & $0.85 \pm 0.01$ & $0.00 \pm 0.0$ \\
\hline $\begin{array}{l}\text { Bouchouk } \\
\text { de Guergour } 1\end{array}$ & $0.0 \pm 0.0$ & $3.81 \pm 0.01$ & $20.14 \pm 0.01$ & $4.51 \pm 0.01$ & $2.20 \pm 0.01$ & $0.4 \pm 0.00$ & $0.0 \pm 0.0$ & $0.60 \pm 0.01$ & $0.05 \pm 0.01$ & $0.04 \pm 0.01$ & $0.53 \pm 0.01$ & $2.1 \pm 0.0$ & $0.08 \pm 0.01$ \\
\hline $\begin{array}{l}\text { Bouchouk } \\
\text { de Guergour } 2\end{array}$ & $0.0 \pm 0.0$ & $4.21 \pm 0.01$ & $14.54 \pm 0.01$ & $2.15 \pm 0.01$ & $1.11 \pm 0.01$ & $0.35 \pm 0.01$ & $0.0 \pm 0.0$ & $0.33 \pm 0.01$ & $0.02 \pm 0.01$ & $0.05 \pm 0.01$ & $0.33 \pm 0.01$ & $1.55 \pm 0.1$ & $0.05 \pm 0.01$ \\
\hline X-Aghenfas1 & $0.0 \pm 0.0$ & $8.71 \pm 0.01$ & $36.30 \pm 0.01$ & $11.78 \pm 0.01$ & $1.47 \pm 0.01$ & $1.03 \pm 0.01$ & $0.0 \pm 0.0$ & $0.86 \pm 0.01$ & $0.09 \pm 0.0$ & $0.06 \pm 0.01$ & $0.00 \pm 0.0$ & $0.00 \pm 0.00$ & $0.06 \pm 0.01$ \\
\hline X-Aghenfas2 & $0.0 \pm 0.0$ & $3.54 \pm 0.01$ & $13.94 \pm 0.01$ & $6.50 \pm 0.01$ & $0.94 \pm 0.01$ & $0.54 \pm 0.01$ & $0.0 \pm 0.0$ & $0.09 \pm 0.01$ & $0.06 \pm 0.01$ & $0.04 \pm 0.01$ & $0.00 \pm 0.0$ & $0.00 \pm 0.0$ & $0.09 \pm 0.00$ \\
\hline Bounguergueb 1 & $0.0 \pm 0.0$ & $1.84 \pm 0.01$ & $13.94 \pm 0.06$ & $4.45 \pm 0.02$ & $0.99 \pm 0.01$ & $0.67 \pm 0.01$ & $0.0 \pm 0.0$ & $1.57 \pm 0.01$ & $0.0 \pm 0.0$ & $0.52 \pm 0.01$ & $0.00 \pm 0.0$ & $0.86 \pm 0.01$ & $0.34 \pm 0.01$ \\
\hline Bounguergueb 2 & $0.0 \pm 0.0$ & $3.46 \pm 0.01$ & $14.13 \pm 0.04$ & $3.25 \pm 0.01$ & $1.06 \pm 0.01$ & $1.03 \pm 0.04$ & $0.0 \pm 0.0$ & $2.82 \pm 0.01$ & $0.0 \pm 0.0$ & $0.62 \pm 0.01$ & $0.00 \pm 0.0$ & $1.15 \pm 0.07$ & $0.66 \pm 0.01$ \\
\hline Ronde de miliana 1 & $0.0 \pm 0.0$ & $1.44 \pm 0.02$ & $16.58 \pm 0.03$ & $4.03 \pm 0.01$ & $1.14 \pm 0.01$ & $1.22 \pm 0.01$ & $0.0 \pm 0.0$ & $0.66 \pm 0.01$ & $0.14 \pm 0.01$ & $0.00 \pm 0.0$ & $1.05 \pm 0.01$ & $0.99 \pm 0.01$ & $0.04 \pm 0.01$ \\
\hline Ronde de miliana 2 & $0.0 \pm 0.0$ & $0.0 \pm 0.0$ & $20.15 \pm 0.01$ & $4.51 \pm 0.01$ & $0.82 \pm 0.01$ & $0.73 \pm 0.01$ & $0.0 \pm 0.0$ & $0.91 \pm 0.01$ & $0.09 \pm 0.01$ & $0.08 \pm 0.01$ & $1.51 \pm 0.01$ & $2.1 \pm 0.00$ & $0.12 \pm 0.01$ \\
\hline Sigoise 1 & $0.0 \pm 0.0$ & $2.57 \pm 0.01$ & $22.45 \pm 0.01$ & $3.31 \pm 0.01$ & $0.63 \pm 0.01$ & $0.25 \pm 0.01$ & $0.0 \pm 0.0$ & $0.33 \pm 0.01$ & $0.27 \pm 0.01$ & $0.00 \pm 0.0$ & $0.34 \pm 0.01$ & $0.77 \pm 0.02$ & $0.00 \pm 0.00$ \\
\hline Sigoise 2 & $0.0 \pm 0.0$ & $1.38 \pm 0.01$ & $27.85 \pm 0.01$ & $2.02 \pm 0.01$ & $0.48 \pm 0.01$ & $0.15 \pm 0.01$ & $0.0 \pm 0.0$ & $0.29 \pm 0.01$ & $0.03 \pm 0.01$ & $0.00 \pm 0.0$ & $0.21 \pm 0.01$ & $0.11 \pm 0.01$ & $0.00 \pm 0.00$ \\
\hline Grosse du Hamma 1 & $0.0 \pm 0.0$ & $9.67 \pm 0.01$ & $28.14 \pm 0.01$ & $3.13 \pm 0.01$ & $4.03 \pm 0.04$ & $0.57 \pm 0.01$ & $0.0 \pm 0.0$ & $0.89 \pm 0.01$ & $0.34 \pm 0.01$ & $0.31 \pm 0.01$ & $0.90 \pm 0.01$ & $1.06 \pm 0.08$ & $0.00 \pm 0.00$ \\
\hline Grosse du Hamma 2 & $0.0 \pm 0.0$ & $14.92 \pm 0.01$ & $32.98 \pm 0.01$ & $2.22 \pm 0.01$ & $3.00 \pm 0.0$ & $0.24 \pm 0.01$ & $0.0 \pm 0.0$ & $0.25 \pm 0.01$ & $0.22 \pm 0.01$ & $0.23 \pm 0.01$ & $0.43 \pm 0.01$ & $0.23 \pm 0.0$ & $0.00 \pm 0.00$ \\
\hline $\begin{array}{l}\text { Rougette } \\
\text { de Guelma } 1\end{array}$ & $0.0 \pm 0.0$ & $3.33 \pm 0.04$ & $18.51 \pm 0.01$ & $4.05 \pm 0.01$ & $2.34 \pm 0.01$ & $0.34 \pm 0.01$ & $0.0 \pm 0.0$ & $1.07 \pm 0.01$ & $0.11 \pm 0.01$ & $0.00 \pm 0.0$ & $1.35 \pm 0.01$ & $0.76 \pm 0.01$ & $0.08 \pm 0.01$ \\
\hline $\begin{array}{l}\text { Rougette } \\
\text { de Guelma } 2\end{array}$ & $0.0 \pm 0.0$ & $3.65 \pm 0.01$ & $14.02 \pm 0.01$ & $10.40 \pm 0.01$ & $1.52 \pm 0.01$ & $0.54 \pm 0.01$ & $0.0 \pm 0.0$ & $0.81 \pm 0.01$ & $0.09 \pm 0.01$ & $0.00 \pm 0.0$ & $0.71 \pm 0.01$ & $2.17 \pm 0.01$ & $0.02 \pm 0.01$ \\
\hline
\end{tabular}

1: Crop year $1 \quad$ 2: Crop year 2 
Table 5

Variance analysis ANOVA: Phenolic compounds

\begin{tabular}{|c|c|c|c|c|c|c|c|c|c|c|c|c|c|}
\hline & $\mathrm{PA}$ & 3,4-DHPEA & Tyr & 4-HBA & CafA & VA & $p-C A$ & $T$ & $\mathrm{FA}$ & $0-C A$ & $O G$ & 3-MBA & $\operatorname{Cin} A$ \\
\hline & $F(p) D D L$ & $F(p) D D L$ & $F(p) D D L$ & $F(p) D D L$ & $F(p) D D L$ & $F(p) D D L$ & $F(p) D D L$ & $F(p) D D L$ & $F(p) D D L$ & $F(p) D D L$ & $F(p) D D L$ & $F(p) D D L$ & $F(p) D D L$ \\
\hline $\begin{array}{l}\text { Population } \\
\text { variety Effect }\end{array}$ & $\begin{array}{c}5.9820 \\
(p<0.001)\end{array}$ & $\begin{array}{c}13.8520 \\
(p \leq 0.001)\end{array}$ & $\begin{array}{c}4.520 \\
(p<0.001)\end{array}$ & $\begin{array}{c}8.8920 \\
(p \leq 0.001)\end{array}$ & $\begin{array}{c}7.9120 \\
(p<0.001)\end{array}$ & $\begin{array}{c}7.3720 \\
(p \leq 0.001)\end{array}$ & $\begin{array}{c}3.9220 \\
(p \leq 0.001)\end{array}$ & $\begin{array}{c}8.5520 \\
(p \leq 0.001)\end{array}$ & $\begin{array}{c}4.3320 \\
p<0.001)\end{array}$ & $\begin{array}{c}12.7620 \\
(p \leq 0.001)\end{array}$ & $\begin{array}{l}21.5120 \\
(p<0.001)\end{array}$ & $\begin{array}{c}4.5620 \\
(p \leq 0.001)\end{array}$ & $\begin{array}{c}19.4520 \\
(p<0.001)\end{array}$ \\
\hline Year Effect & $\begin{array}{c}3.251 \\
(p>0.05)\end{array}$ & $\begin{array}{c}1.031 \\
(p>0.05)\end{array}$ & $\begin{array}{c}0.461 \\
(p>0.05)\end{array}$ & $\begin{array}{c}0.421 \\
(p>0.05)\end{array}$ & $\begin{array}{c}0.691 \\
(p>0.05)\end{array}$ & $\begin{array}{c}0.861 \\
(p>0.05)\end{array}$ & $\begin{array}{c}1.671 \\
(p>0.05)\end{array}$ & $\begin{array}{c}0.221 \\
(p>0.05)\end{array}$ & $\begin{array}{c}5.931 \\
(p \leq 0.01)\end{array}$ & $\begin{array}{c}3.321 \\
(p>0.05)\end{array}$ & $\begin{array}{c}0.261 \\
(p>0.05)\end{array}$ & $\begin{array}{c}0.0031 \\
(p>0.05)\end{array}$ & $\begin{array}{c}0.661 \\
(p>0.05)\end{array}$ \\
\hline
\end{tabular}

Significance level at $\mathrm{P} \leq 0.001 \mathrm{HS} ; \mathrm{P} \leq 0.01 \mathrm{MS} ; \mathrm{P} \leq 0.05 \mathrm{~S} ; \mathrm{P} \geq 0.05 \mathrm{NS}$.

Projection of variables of the factorial plan ( $\left.\begin{array}{ll}1 \times & 2\end{array}\right)$

Active and additional Variables

*Additional Variable

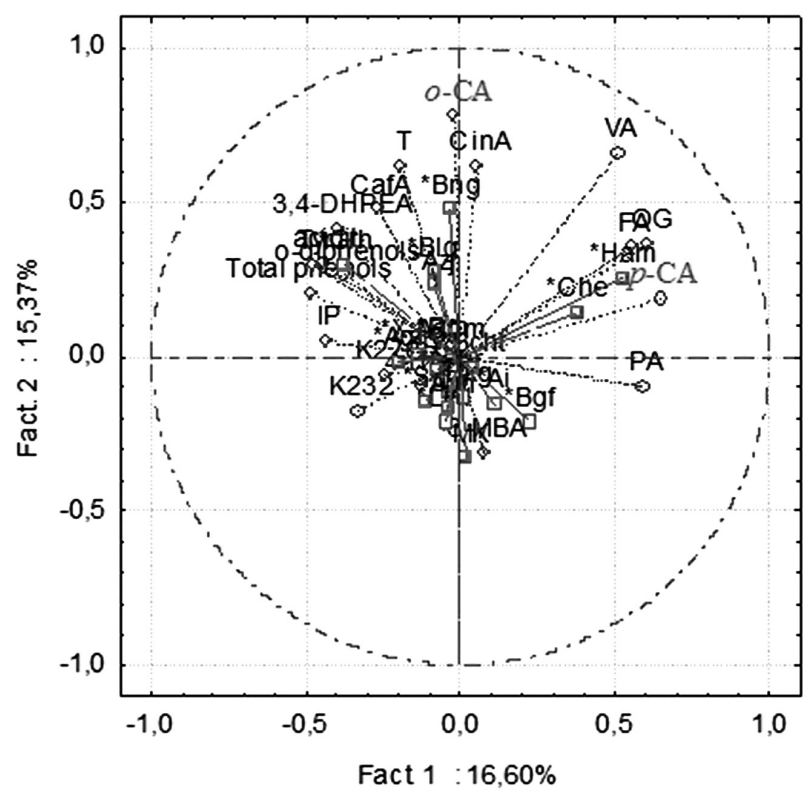

Figure 1

Circle of correlation between the quantitative variables on the factorial planes 1-2

GalA: Gallic acid ; PA: Protocatechuic acid ; 3,4-DHPEA: 3,4-di hydroxyphénylethanol acid or hydroxytyrosol ; Tyr: Tyrosol;

4-HBA: 4-hydroxybenzoic acid ;CafA: Caffeic acid; VA: Vanillic acid; $p$-CA: $p$-coumaric acid; FA: Ferulic acid or

trans-4-hydroxy-3-methoxycinnamic acid, o-CA: o-coumaric acid; 3-MBA: 3-methoxybenzoic acid; CinA: Cinnamic acid;

T: Taxifolin ;OG: Oleuropein; IP: peroxide value.

Chemlal (Che); Azeradj (Azj) and Sigoise (Sg): Mekki (Mk); Neb djemel (Nd); Hamra (Ham); Blanquette de Guelma (Blg); Aberkane (Abr); Aimel (Ai); Rougette de la Mitidja (Rm);

Aguenaou (Agn); Boughenfas (Bgf); Bouichret (Bcht); Aghenfas (Agf); Bouchouk de Guergour (Bkg), X-Aghenfas (X-Agf), Bounguergueb (Bng), Ronde de Miliana (Rdm), Limli (Lim), Grosse du Hamma (Grh), Rougette de Guelma (Rg).

are reflected in axis 2Tyrosol, taxifolin, ferulic acid and cinnamic acid are reflected in axis 3 .

The confrontation between the correlations' circle of variables and the individual projections (Fig. 2) allowed for determining the characteristics of individuals according to their characteristics whether physico-chemical or biochemical, in relation with the plane axis. Thus, axis 2 allowed for pointing out that the individuals with large fruits (Grosse du Hamma, Aghenfas, Aberkane, Rougette de Guelma...) were clearly distinct

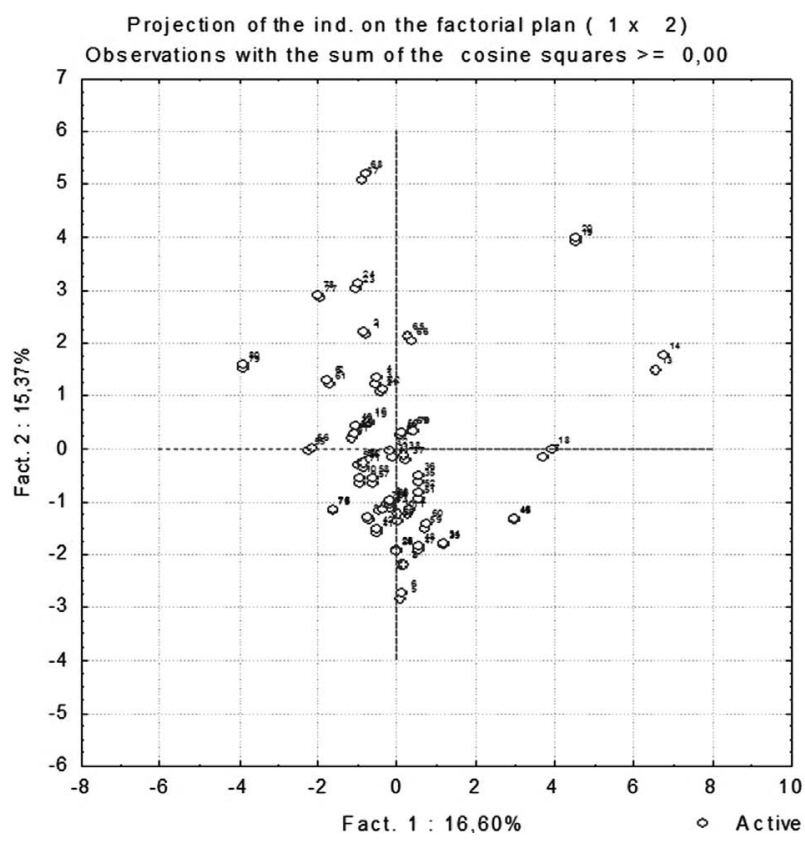

Figure 2

Projection of the individuals according to the different principal components as plane of projection of the axis 1-2.

from the others, with the highest rates of tyrosol, hydroxytyrosol, 4-HBA and total phenols. The individuals with medium-sized fruits were very close to the individuals with large fruits, with high rates of hydroxytyrosol, cinnamic acid and taxifolin (Ronde de Miliana, Rougette de la Mitidja, Bounguergueb...). The individuals with small fruits (Chemlal, Hamra, Mekki ...) have the highest levels of oleuropein, vanillic acid, protocatechuic acid, $p$-coumaric acid and ferulic acid.

With regards to the representation (Fig. 3), the most important was group $\mathrm{V}$ which has the majority of the studied varieties with the exception of three lower groups which were clearly distinguishable in relation to the whole, particularly Chemlal, Boughenfas and Hamra (variety with large fruits); probably due to parental relation. These three lower groups took positive values in relation to group I, group II, group III and group IV. They were the varieties with large fruits (Grosse du Hamma) from year 1 and year 2, Rougette de Guelma from year 1, Sigoise from year 1; and with medium-sized fruits (Ronde de Miliana from year 2 and Blanquette de Guelma from year 1). The results found by AHC 


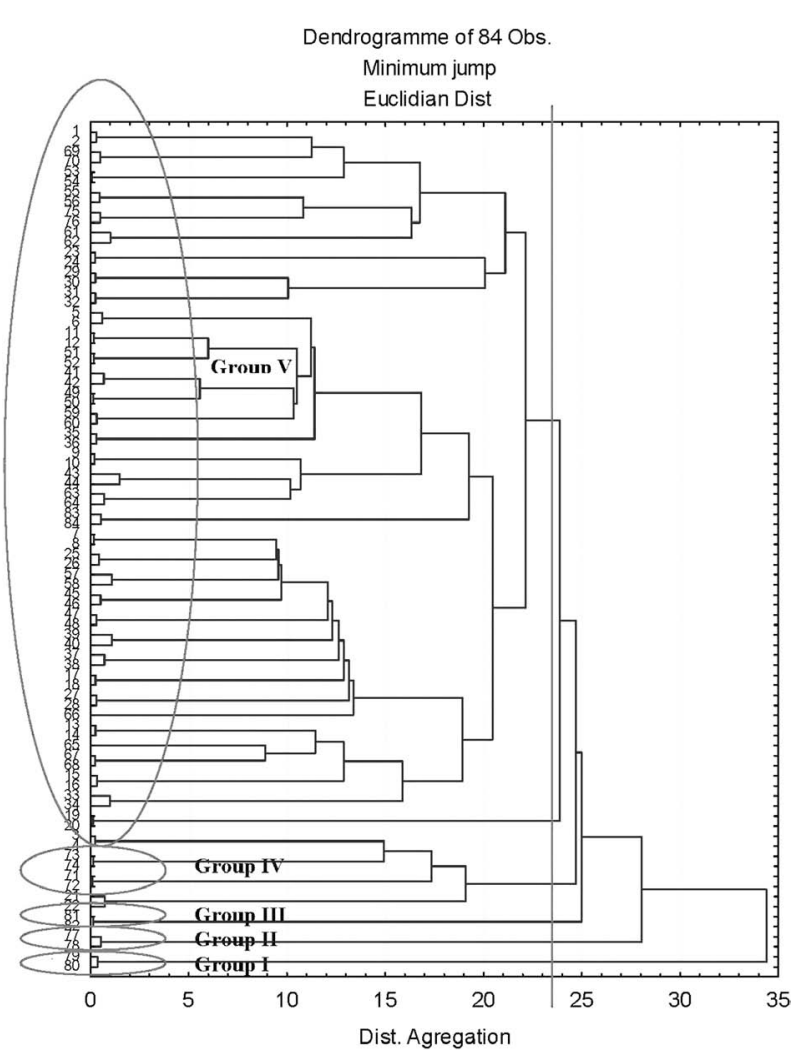

Figure 3

Dendrogram of classification of oil of the different varieties studied.

had the same structure in all varieties as those revealed by APC and the Test of Newman-Keuls for homogenization.

\section{CONCLUSIONS}

The variety effect exerted an important influence while the year effect was negligible, knowing that the Olivier's collection was taken as reference to confirm our hypotheses (the varieties were in the same climatic and pedological conditions). It seemed that the phenolic compounds could be considered as the biochemical marker of varieties' characteristics.

In Algerian traditional oleoculture, the varieties with large and medium-sied fruits are often devoted to canning and the small fruits are destined for oil production. The first could serve for eventual blending in order to correct the chemical composition of the oil from varieties with small fruits and weak content in phenolic compounds.

Consequently, in order to improve the quality of our oil and to enhance the value of the phytogenetic resources, these local population varieties which could be considered secondary (Grosse du Hamma, Aghenfas, Azeradj, Aguenaou, Aberkane, Bouchouk de Guergour, X-Aghenfas, Rougette de Guelma, Sigoise) could be given more priority for the new olive plantations included in a project which is underway for the plantation of one million hectare (MADR. 2010) and to use them instead of the old olive varieties.

\section{ACKNOWLEDGEMENTS}

This work was partially supported by the Algerian Ministry of Higher Education and Scientific Research. The authors are thankful to the farm pilot of the ITAFV for the vegetable material. The authors also wish to thank Bendini. A (department of food science, University of Bologna, Via Ravennate 1020, Cesena, 47023, Italy) for method analysis, and other aids. Special thanks go to Professor Guermouche M-H of the Chemical faculty of Algiers's University for his precious chromatographic advice.

\section{REFERENCES}

Akasbi M, Shoeman DW, Saari Csallany A. 1993. High performance liquid chromatography of selected phenolic compounds in olive oil. J. Am. Oil Chem. Soc. 70, 367-370.

Amiot MJ, Fleuriet A, Macheix J J. 1986. Importance and evolution of phenolic compounds in olive during growth and maturation. J. Agric. Food Chem. 34, 823826.

Angerosa F, D'Alessandro N, Konstantinou P, DiGiacinto L. 1995. GC-MS Evaluation of phenolic compounds in virgin olive oil. J. Agric. Food Chem. 43, 1802-1807.

Artajo LS, Romero MP, Suárez M, Motilva MJ. 2007. Partition of phenolic compounds during the virgin olive oil industrial extraction process. Eur. Food Res. Technol. 225, 617-625.

Baccouri O, Guerfel M, Baccouri B, Cerretani L, Bendini A, Lercker G, Zarrouk M, Daoud Ben Miled D. 2008. Chemical composition and oxidative stability of Tunisian monovarietal virgin olive oils with regard to fruit ripening. Food Chem. 109, 743-754.

Beltrán G, Jiménez A, Aguilera MP, Uceda M. 2000. Phenolic fraction analysis by HPLC of Arbequina virgin olive oils. Relationship with bitterness K225 and oil stability. Grasas Aceites, 51, 320-324.

Beltrán G, Paz Aguilera M, Del Rio C, Sánchez S, Martinez L. 2005. Influence of fruit ripening process on the natural antioxidant content of Hojiblanca virgin olive oils. Food Chem. 89, 207-215

Bendini A, Bonoli M, Cerretani L, Biguzzi B, Lercker G, Toschi TG. 2003. Liquid-liquid and solid- phase extractions of phenols from virgin olive oil and their separation by chromatographic and electrophoretic methods. J. Chromatogr. A, 985,424-433.

Bonoli M, Bendini A, Cerretani L, Lercker G, Toschi TG. 2004.Qualitative and semiquantitative analysis of phenolic compounds in extra virgin olive oils as a function of the ripening degree of olive fruits by different analytical techniques. J. Agric. Food Chem. 52, 7026-32

Boukachabine N, Kartas A, Ajana H, El Antari A. 2007. Evolution des polyphénols totaux et de l'amertume de l'huile au cours de la maturité des olives de la variété Picholine marocaine. Olivæ. 107, 29-33.

Cortesi N,Azzolini M, Rovellini P, Fedeli E. 1995. I componenti minori polari degli oli vergini di oliva: ipotesi di struttura mediante LC-MS. Riv. Ital. Sostanze Gr. 72, 241.

Dugo G, Turco V, Pollicino D, Mavrogeni E, Pipitone F. 2004. Caractérisation d'huiles d'olives vierges siciliennes. Variation qualitative des huiles des fruits des cultivars 'Biancolilla', 'Nocellara del Belice', 
'Cerasuola', 'Tonda Iblea' et'Crastu' en fonction des techniques et de l'époque de récolte des olives. Olivae, 101, 44-52.

EC (1991). Commission Regulation (EEC) $n^{\circ}$ 2568/91 on the Characteristics of Olive Oil and Olive Residue Oil and the Relevant Methods of Analysis. Official Journal of the European Community $n^{\circ}$ L248.

Forcadell M L, Servili M, Comax M, Miguel X, De la Torre MC. 1987. Détermination du tyrosol et de I'hydroxytyrosol dans les huiles vierges d'olive. Ver. Franc. Corps Gras. 34, 547.

Gimeno E, Fitc M, Lamuela-Ravent s R M, Farré M 2002. Effect of ingestion of virgin olive oil on human low-density lipoprotein composition. Eur. J. Clin. Nutr. 56, 114-120.

Gutiérrez-Rosales F, Arnaud T, Garrido A. 2001. Contribution of polyphenols to the oxidative stability of virgin olive oil. J. Sci. Food Agric. 81, 1463-1470.

Gutiérrez-Rosales F, R os J J, Gómez-Rey L. 2003. Main polyphenols in the bitter Taste of virgin olive oil. Structural confirmation by on-line high-performance liquid chromatography electrospray ionisation mass spectrometry. J. Agric. Food Chem. 51, 6021-6025.

Litridou M, Linssen J, Schols H, Bergmans M, Posthumus M,Tsimidou M, Boskou D. 1997. Phenolic compounds in virgin olive oils: Fractionation by solid phase extraction and antioxidant activity assessment. J. Sci. Food Agric. 74, 169-174.

Ministry for Agriculture and the Rural development (M.A.D.R) 2010. Report of the ministry for agriculture and the rural development. Program development of oleiculture 2010/2014.

Mateos R, Espartero JL, Trujillo M, Ríos JJ, LeónCamacho, Alcudia F, Cert A. 2001. Determination of phenols, flavones and lignans in virgin olive oils by solid-phase extraction and high-performance liquid chromatography with diode array ultraviolet detection. J. Agric. Food Chem. 40, 1577-1580.

Mateos R, Trujillo M, Pérez-Camino C, Moreda W, Cert A. 2005. Relationships between oxidative stability, triacylglycerol composition, and antioxidant content in olive oil matrices. J. Agric. Food Chem. 53, 5766-5771.

Montedoro G, Servili M, Baldioli M, Miniati E. 1992. Simple and hydrolyzable phenolic compounds in virgin olive oil. 2. Simple charactérization of the hydrolyzable fraction. J. Agric. Food Chem. 49, 2185-2192.

Oliveras-López MJ, Innocenti M, Giaccherini C, leri F, Romani A, Mulinacci N. 2007. Study of phenolic composition of spanish and italian monocultivar extra olive oils : Distribution of lignans, secoiridoidic, simple phenols and flavonoids. Talanta 73, 726-732.

Ollivier D, Pinatel C, Dupuy N, Guérère M, Artaud J. 2007. Caractérisations sensorielles et chimiques d'huiles d'olive vierges de six AOC françaises. Rev. OCL. 14, 116-129.

Pellegrini N, Visioli F, Buratti S, Brighenti F. 2001. Direct analysis of total antioxydant activity of olive oil and studies on the influence of heating. J. Agric. Food Chem. 49, 2532-2538.

Pinelli P, Galardi C, Mulinacci N, Vincieri F F, Cimato A, Romani A. 2003. Minor polar compound and fatty acid analyses in monocultivar virgin olive oils from Tuscany. Food Chem. 80, 331-336.

Ryan D, Robards K, Lavee S.1998. Evaluation of the quality of the olive oil. Olivæ 75, 1-6.

Saitta M,Lo Curto S, Salvo F, Di Bella G, Dugo G. 2002. Gas chromatographic-tandem mass spectrometric identification of phenolic compounds in Sicilian olive oils. Anal. Chim. Acta, 466, 335-344.

Singleton VL, Rossi JA. 1965. Colorimetry of total phenolics with phosphomolibdic-phosphotungstic acid reagents. Am. J. Enol. Vitic. 16, 144-158.

Tamendjari A, Angerosa F, Mettouchi S et Bellal MM. 2009a. The effect of fly attack (Bactrocera oleae). On the quality and phenolic content of Chemlal olive oil. Grasas Aceites, 60, 509- 515.

Tamendjari A, Sahnoune M, Mettouchi S et Angerosa F. 2009b. Effet de l'attaque du ravageur Bactrocera oleae sur la qualité de l'huile d'olive de trois variétés algériennes: Chemlal, Azzeradj et Bouchouk. Riv. Ital. Sostanze Gr. 86, 103-111.

Tamendjari A, Laribi R, Bellal MM. 2011. Effet de l'attaque des olives par Bactrocera oleae sur la qualité et la fraction volatile de l'huile de deux variétés algériennes. Riv. Ital. Sostanze Grasse. 88, 118-127.

The International Olive Oil Council. IOOC 2009. Détermination of des biophénols des huiles d'olive par HPLC. COI/T.20/Doc n 29.

Tsimidou M, Papadopoulos G, Boskou D. 1992. Determination of phenolic compounds in Virgin olive oil by reversed-phase HPLC with emphasis on UV detection. Food Chem. 44, 53-60.

van der Sluis A, Dekker N, Van Boekel. 2005. Activity and concentration of polyphenolic antioxidants in apple juice 3. Stability during storage J. Agric. Food Chem. 53, 1073-1080.

Veillet S, Tomao V, Bonard I, Ruiz K, Chemat F. 2009. Chemical changes in virgin olive oils a function of crushing systems: Stone mil land hammer crusher. C.R. Chimie 12, 895-904.

Visioli F, Poli A, Galli C. 2002. Antioxidant and other biological activities of phenols from olives and olive oil. Med. Res. Rev. 22, 65-7.

Recibido: $17 / 7 / 12$ Aceptado: 29/1/13 